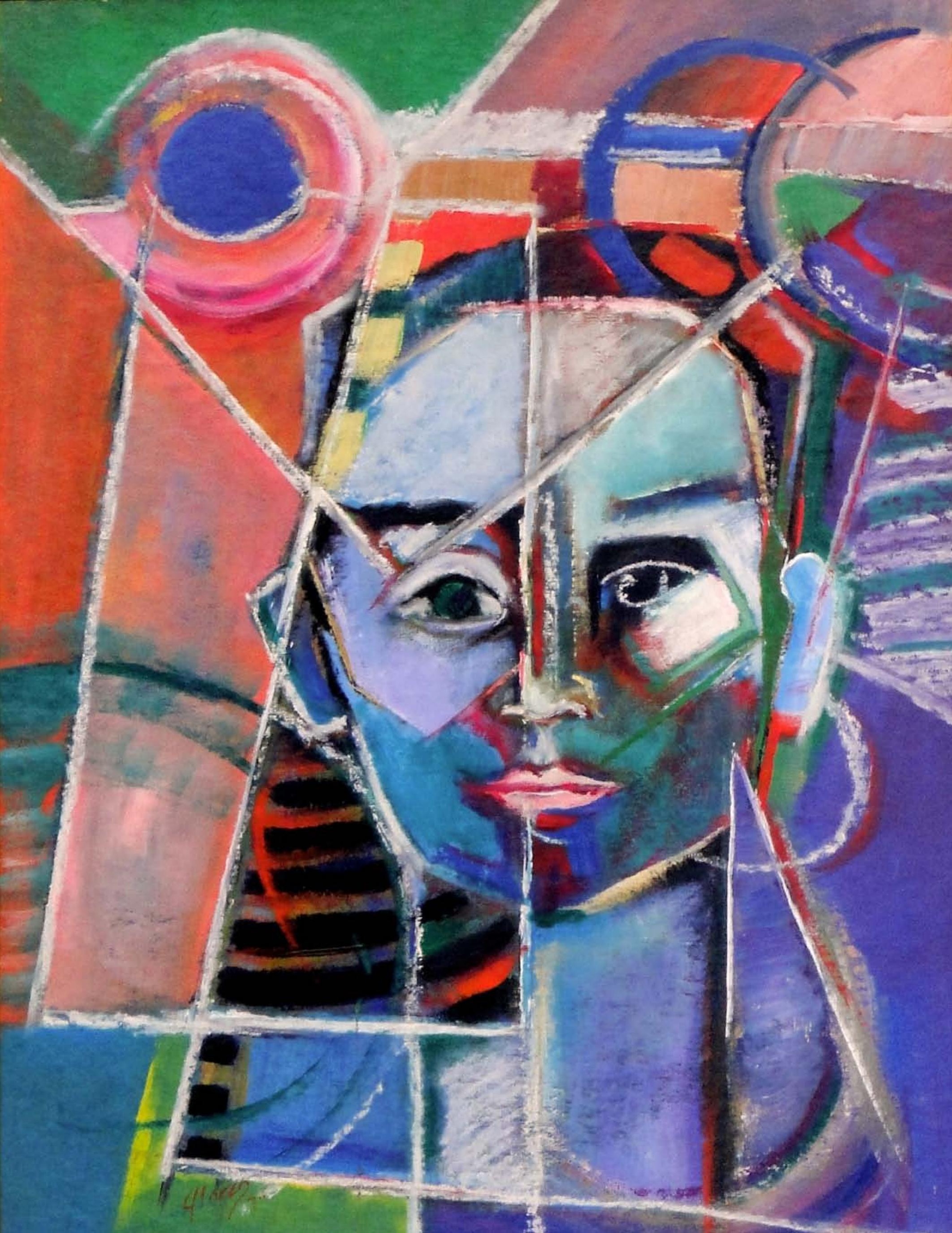




\section{La violencia en el Distrito Central desde la óptica del enfoque estructural y criminal}

Marysabel Zelaya OchoA*

RESUMEN. La violencia urbana ha existido desde que existe la ciudad, sin embargo, en los últimos años se ha convertido en tema de gran relevancia debido a las nuevas formas que ha asumido y al incremento alarmante de su magnitud, expresión clara de la crisis urbana en la ciudades, de las cuales el Distrito Central no es la excepción. Este articulo analiza la violencia en el Distrito Central desde dos enfoques: violencia estructural y violencia criminal; el primero considera que la violencia tiene origen en la pobreza, las desigualdades económicas, la exclusión, segregación social y la falta de oportunidades sociales, políticas y económicas generadas por el sistema. El segundo enfoque pone de relieve que la violencia criminal en el DC se manifiesta en el crecimiento de los niveles de victimización, que adopta formas como robo, asaltos, homicidios, sicariato y secuestro, entre los delitos de mayor trascendencia.

Palabras claves: violencia, pobreza, exclusión social, violencia estructural, violencia criminal.

SUMMARY. Urban violence has existed since the city exists, however, in recent years has become a topic of great importance due to new forms it has assumed and due to the alarming increase of its magnitude, clear expression of the urban crisis in the cities, of which the Central District is no exception. This article analyzes the violence in the Central District from two perspectives: structural violence and criminal violence; the first considers that violence is rooted in poverty, economic inequality, exclusion, social segregation and lack of social, political and economic opportunities, generated by the system. The second approach emphasizes that criminal violence in DC manifests in the increasing levels of victimization, which takes forms such as theft, assaults, murders, killings and kidnapping amongst the crimes of greater significance.

Keywords: violence, poverty, social exclusion, structural violence, criminal violence.

\section{Introducción}

En Honduras el problema de la violencia se ha agravado en los últimos años; a diario los medios de comunicación muestran como esta situación azota la población poniendo en riesgo su bienestar físico emocional y mental.

La palabra violencia se emplea para referirse a un conjunto de situaciones heterogéneas. El término se usa para enunciar hechos como el intercambio agresivo de palabras, situaciones de maltrato físico y psicológico, el irrespeto a la propiedad privada manifestada a través del robo y hurto a los bienes, homicidios, asesinatos, acciones de narcotráfico y secuestro, hechos cada vez más frecuentes en la sociedad actual.

La evidencia más notoria de la violencia en Honduras se manifiesta en los principales centros urbanos del país, el Distrito Central y San Pedro Sula. El crecimiento de los índices de violencia en estas ciudades quizá esté relacionado con el hecho que gran parte de la población se concentra en estos municipios (aproximadamente el 23\% del total poblacional para el 2010) lo que genera mayor demanda de servicios públicos, fuentes de empleo, viviendas, congestionamiento vial, falta de espacios recreativos. Todas estas demandas generan una problemática social que en muchas ocasiones se desencadena en violencia ciudadana.

El incremento de la violencia urbana está ligado a factores como la pobreza, la desigualdad social, el crecimiento acelerado y desordenado de las ciudades, el uso de armas, el consumo de alcohol y otras drogas y las acciones conexas al narcotráfico.

Recibido: mayo de 2011 /Aceptado y versión final agosto de 2011.

* Dra. en Ciencias Sociales. marysabelzo@yahoo.com 
El análisis que se presenta se hace teniendo en cuenta el enfoque estructural de la violencia ligado sobre todo a las condiciones de pobreza, desigualdad y exclusión social y el enfoque criminal que explica las posibles razones que conducen a cometer un delito.

La investigación tiene como objetivo general analizar las condiciones de violencia estructural y violencia criminal que presentó el Distrito Central en el periodo 2002-2009, los datos muestran una panorámica general del contexto y permiten reflexionar respecto al impacto que estas condiciones generan en la población capitalina.

\section{Metodología}

Este artículo surge del contexto de la investigación de tesis doctoral "La Crisis de los Espacios Públicos Urbanos en el Distrito Central”, la investigación se desarrolló bajo un enfoque mixto implicando un proceso de recolección, análisis y vinculación de datos cualitativos y cuantitativos cuyo propósito estuvo encaminado a responder al planteamiento de la investigación propuesta.

Se hizo uso de datos secundarios de las Encuestas Permanentes de Hogares (EPHPM) en una serie de 8 años (2002-2009); los datos de las EPHPM sirvieron de fundamento para analizar la situación de violencia estructural en el dominio del Distrito Central a partir del método de Necesidades Básicas Insatisfechas (MNBI) y del Método de Línea de Pobreza (LP).

En el contexto de la violencia criminal se hizo uso de los datos generados por el Observatorio de la Violencia del Distrito Central en el periodo 2007-2009, publicados a través de boletines, a nivel nacional y del Distrito Central. La información presentada por el Observatorio parte de los datos registrados por la Policía Preventiva, la Dirección General de Investigación Criminal (DGIC) y Medicina Forense.

\section{Contexto teórico de la investigación}

El estudio de la violencia puede tener muchas aristas de análisis, sin embargo, en el contexto de esta investi- gación se analiza teniendo en cuenta dos enfoques: la violencia estructural y la violencia criminal.

\subsection{Enfoque de violencia estructural}

Uno de los enfoques que permite explicar la violencia urbana parte de una postura de origen estructural que se ha ido gestando paso a paso ante la necesidad de explicar que la violencia tiene origen en la pobreza, las desigualdades económicas, la exclusión, segregación social y la falta de oportunidades sociales, políticas y económicas generadas por el sistema capitalista.

El desarrollo teórico del enfoque de violencia estructural se gestó poco a poco, ante la necesidad de explicar las interacciones de las prácticas violentas en los diversos ámbitos sociales.

La violencia estructural tiene un precedente significativo en las explicaciones que los teóricos marxistas daban a la explotación y la marginación de los trabajadores. Otro precedente más cercano está en los años sesenta cuando Martin Luther King, líder no violento de los negros norteamericanos en su lucha contra el racismo, contribuyó a entrever causas más profundas de la marginación en algunos de sus escritos. Sin embargo, ha sido el investigador para la paz Johan Galtung quién más ha desarrollado la temática, explicando como la violencia estructural que englobaría a la pobreza condicionada estructuralmente (cuando no estuviera garantizado el acceso a bienes como alimentos, agua, vestido, vivienda, medicamentos y escolaridad), a la represión política (cuando se vulnere derechos como los relativos a la libertad de expresión, de reunión, de movimiento, de protección jurídica, de movilización, de formación de la conciencia, al trabajo...), y a la alienación (cuando hubiera obstáculos, evitables, a la satisfacción de necesidades tales como la de comprender las condiciones de la propia existencia, de comunidad, de compañerismo, de amistad, de solidaridad, de alegría, de dar significados a la propia vida, de tener algún tipo de comunicación con la naturaleza).

(Jiménez B , Muñoz, A 2004: 1227).

Partiendo del enfoque de Galtung (1990), la violencia estructural es originada por la injusticia y la desigualdad 
como consecuencia de la propia estructura social y tiende a manifestarse como violencia indirecta.

Durante la edad media los pobres eran vistos como algo natural y asimismo era ayudarlos. La Ley de los Pobres de la época daba importancia a dos aspectos: la alta responsabilidad social que se tenía por los pobres en donde ayudar a estos era una obligación al igual que mantenerlos bajo control. En relación al primer aspecto los pobres eran vistos como algo natural y así mismo era ayudarlos, sin embargo, esta ley de responsabilidad social por los pobres llega a su fin con la Revolución Industrial así se tiene la idea de que la pobreza es un problema individual, pero también un problema social, pero no porque le corresponda a éste solucionarlo sino porque incide negativamente en el funcionamiento de un sistema que se ha consolidado: el capitalismo (Luna, V 2005: 8,9).

Bajo ese contexto histórico puede entenderse como la pobreza se consolida dentro del modo de producción capitalista puesto que el interés de ayudar a los pobres no está centrado en elevar su nivel de bienestar sino en mantener intacto el sistema capitalista. He aquí la explicación del porqué después de tantos programas y proyectos destinados a reducir los niveles de pobreza, los pobres siguen estando ahí más vigentes que nunca.

\subsection{Enfoque de violencia criminal}

Según el informe de Violencia y Salud elaborado por el Banco Mundial (2002) el siglo XX se recordará como un siglo marcado por la violencia. Honduras no escapa del contexto de la violencia mundial; sus formas de expresión como efecto inmediato han fragmentado el tejido comunitario y amenaza la vida, la salud, la tranquilidad y el bienestar general de la población.

El problema de la violencia criminal ha sido enfocado desde diversas áreas del saber humano entre los que resalta el enfoque sociológico y el enfoque económico.

El enfoque sociológico considera que las fuerzas sociales determinantes del nivel de delincuencia en un país están asociados con las desigualdades del ingreso y riqueza, altos índices de pobreza, ausencia de oportunidades laborales que se manifiestan en altas tasas de desempleo.
Bajo ésta perspectiva se considera que la delincuencia descansa en situaciones de necesidad y carencia.

Desde el enfoque económico Becker (1964) plantea que la opción por infringir la ley de un individuo está condicionada por tres elementos: el beneficio que daría el infringir la ley, el costo o sanción de ser sorprendido o capturado por las autoridades, y la probabilidad de que las autoridades en efecto lo capturen.

Según Becker un individuo hace un análisis costo beneficio de los pro y los contra de violar la ley, y finalmente opta por la decisión de infringir la ley si advierte que son mayores los beneficios. Entonces la probabilidad que se cometa un delito depende de la evaluación por parte del agresor del beneficio esperado y del costo de cometerlo, el que involucra la probabilidad de captura y condena y de la severidad de la sanción en tiempo y dinero.

\section{Resultados de investigación}

La violencia e inseguridad ha estado presente en nuestro país casi de manera permanente, sin embargo, en los últimos diez años se ha presentado un vertiginoso crecimiento de delitos bajo diversas modalidades. En este apartado se presentan los principales resultados de la investigación en relación a la violencia estructural y criminal.

\subsection{Violencia estructural en el Distrito Central}

$\mathrm{Al}$ analizar las condiciones de violencia estructural en el Distrito Central se tiene en cuenta el tema de la pobreza como fenómeno persistente en el municipio, en donde el MNBI y la LP, permiten conocer el comportamiento de los indicadores de pobreza en el contexto del Distrito Central.

\subsection{La pobreza en el Distrito Central desde el método de Necesidades Básicas Insatisfechas \\ El MNBI identifica carencias críticas en una pobla- ción, permite caracterizar la pobreza a través de indicado- res en cuatro áreas de necesidades básicas de las personas: vivienda, acceso a servicios sanitarios, educación básica e ingreso.}


Para Galtung analizar la pobreza partiendo del MNBI, se contextualiza en lo que él llama violencia estructural o indirecta. Este enfoque examina la pobreza como forma de exclusión social teniendo en cuenta la insatisfacción de las necesidades básicas.

En el contexto de las Necesidades Básicas Insatisfechas (NBI), la EPHPM permite presentar una tendencia del comportamiento de la pobreza para los dominios urbanos. ${ }^{1} \mathrm{Al}$ analizar el tema de la pobreza en el área urbana del Distrito Central para el período 2002-2009 puede observarse que más de un tercio de las viviendas presentó carencias en alguno de los servicios básicos, en este sentido, en promedio el $35.9 \%$ de la población en el período 2002-2009 adolecía de la satisfacción de una ó más necesidades básicas. Aunque las cifras en relación a las NBI muestran una tendencia a una leve disminución de la pobreza, sin embargo persisten limitaciones sobre todo en relación al acceso a agua potable y alcantarillado sanitario (Ver gráfico No.1).

En otras palabras, más de un tercio de la población del Distrito Central ha sufrido violencia estructural indirecta hecho que se refleja en el deterioro de las condiciones de vida producto en alguna medida del impacto de modelos económicos neoliberales que conllevan efectos

\section{Gráfico No. 1. Dominio del Distrito Central: necesidades} básicas insatisfechas, en porcentaje 2002-2009

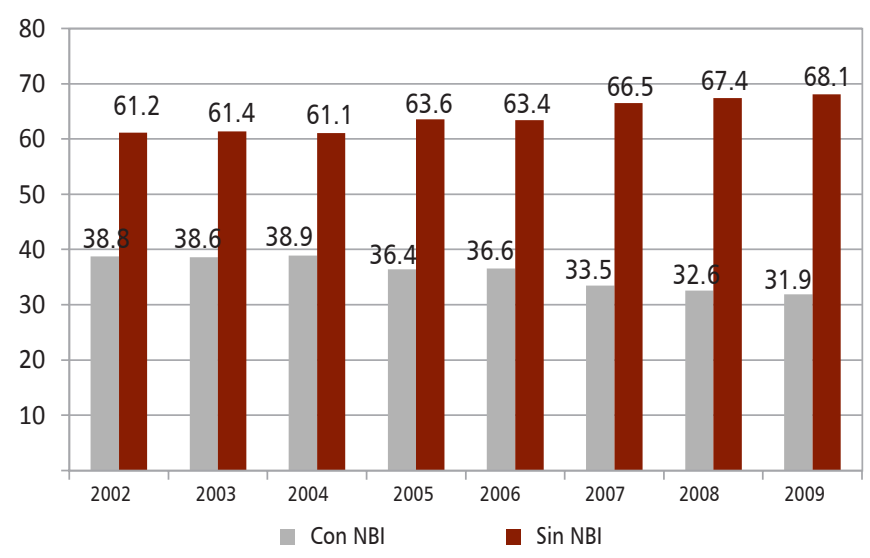

Fuente: Elaboración propia en base a datos de la EPHPM 2004-2009, INE. negativos sobre la inversión del Estado en gastos sociales y sobre el mercado de empleo urbano, en particular para los trabajadores de menor calificación que deben aceptar ocupaciones precarias, informales con baja remuneración. Esta situación brinda limitadas opciones en el mejoramiento de su vivienda y en general de su nivel de bienestar.

En la medida que la población adolece de dos ó más necesidades básicas implica que la magnitud de la pobreza es mayor para este grupo poblacional, en tal sentido, en promedio, el 10.7\% de la población estaba en esta situación, es decir, casi la décima parte de la población vivía en condiciones de pobreza y pobreza extrema. Este porcentaje refleja las carencias de la población del Distrito Central que están directamente relacionadas con la calidad de vida de las familias (Ver gráfico No. 2).

Los sectores pobres de la ciudad viven condiciones de vida precarias evidenciadas en el aumento de desigualdades sociales, acceso y calidad diferenciada de los bienes y servicios de la ciudad lo que está directamente relacionado con el nivel socioeconómico que determina las características de su residencia y de su vecindario.

\section{Gráfico No. 2. Dominio del Distrito Central: viviendas con necesidades básicas insatisfechas en porcentaje, 2002- 2009}

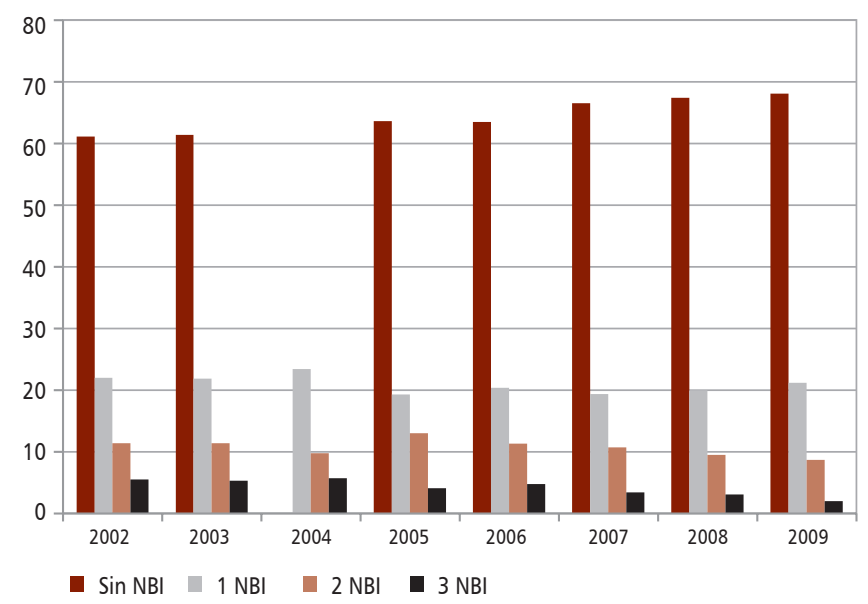

Fuente: Elaboración propia en base a datos de la EPHPM 2002-2009 INE.

1. Área o zona geográfica que se establece teniendo en cuenta el grado de ruralidad o urbanidad. La EPHPM identifica cinco dominios: área urbana, área rural, Distrito Central, San Pedro Sula y el resto urbano. 
Incluso algunos especialistas como Sabatini (1999) han destacado que la importancia del factor socioeconómico y de las inequidades existentes en las sociedades trae consigo polarización y exclusión social. Esta situación fractura de manera profunda la sociedad creando un ambiente propicio para la generación de conflictos sociales cuyas consecuencias afectan el plano personal dando lugar al crecimiento de sentimientos de angustias, miedo, tristeza, odio, violencia y agresión física o verbal, entre otros.

\subsection{La pobreza en el Distrito Central desde el método} Línea de Pobreza

Al utilizar el método de la Línea de Pobreza, la medición se concentra en los ingresos de los hogares fijada por medio del cálculo del costo de una canasta básica. Los hogares que tienen ingresos por debajo de ella se consideran pobres no extremos, y los que tienen ingresos por debajo de una canasta básica de alimentos, pobres extremos.

Datos de la EPHPM registrados en el período 2002. 2009 en el dominio del Distrito Central presentan en promedio, más de la mitad de la población en condición de pobreza evidenciando que este es un problema persistente en la capital.

La pobreza tiende a manifestarse más como pobreza relativa que extrema ${ }^{2}$, sin embargo, en la realidad las diferencias no son extremadamente significativas, en otras palabras, los pobres extremos se manejan bajo una economía de subsistencia, su pobreza se manifiesta en inseguridad alimentaria, problemas de salud sobre todo los relacionados a la desnutrición que afectan de manera directa a la niñez; en el caso de los pobres relativos aunque logran cubrir su canasta básica viven al día, por lo que difícilmente logran superar su condición (Ver cuadro No. 1).
Cuadro No. 1. Distrito Central: personas que viven en hogares por nivel de pobreza 2002-2009

\begin{tabular}{|c|c|c|c|c|c|}
\hline \multirow{2}{*}{ Años } & Total & No pobres & \multicolumn{3}{|c|}{ Pobres } \\
\cline { 4 - 6 } & & & Total & Relativa & Extrema \\
\hline 2002 & 100 & 43.8 & 56.2 & 31.3 & 24.8 \\
\hline 2003 & 100 & 43.6 & 56.3 & 34.5 & 21.7 \\
\hline 2004 & 100 & 43.6 & 56.4 & 31.4 & 25.0 \\
\hline 2005 & 100 & 41.4 & 58.6 & 32.7 & 26.0 \\
\hline 2006 & 100 & 47.7 & 52.9 & 31.7 & 21.1 \\
\hline 2007 & 100 & 44.1 & 55.9 & 40.3 & 15.6 \\
\hline 2008 & 100 & 47.0 & 53.0 & 33.2 & 19.8 \\
\hline 2009 & 100 & 52.3 & 47.7 & 32.3 & 15.4 \\
\hline
\end{tabular}

Fuente: Elaboración propia en base a datos de la EPHPM, INE 2002- 2009.

Uno de los principales problemas del mercado laboral hondureño es el desempleo, situación que afecta todos los sectores de la población siendo muy evidente en el área urbana que concentra tres cuartas partes de la población desocupada del país.

La falta de empleo incide directamente en la evolución de la pobreza, ya que la integración al mercado laboral se postula como una fuerte determinante para estar sobre o debajo de la línea de la pobreza.

Cifras de la EPHPM de mayo del 2009, muestran que el $73 \%$ de las personas desocupadas se ubicaban en las áreas urbanas del país, el Distrito Central al concentrar 14\% de la población total del país (2009), registró para ese año un desempleo abierto ${ }^{3}$ del 7\%, al considerar esta cifra en relación a la cantidad de hogares con personas dependientes el número tiende a aumentar considerablemente. Entre la población desocupada la mayor parte se encontraba entre el rango de 15 a 30 años; población bastante joven con muchas necesidades pendientes de resolver.

Aunque la situación de desempleo en el municipio del Distrito Central es preocupante, pero de igual manera lo es el elevado nivel de empleo precario que no cubre las necesidades ni las expectativas de buena parte de la pobla-

2. Pobreza Extrema: Hogares que tienen un ingreso per cápita inferior al costo de la canasta básica de alimentos Pobreza Relativa: Hogares cuyos ingresos son menores que el costo de la canasta básica y mayor que el costo de la canasta básica de alimentos

3. Desempleo Abierto: personas que quieren trabajar y no encuentran trabajo. 
ción, esta situación se relaciona con el subempleo visible e invisible ${ }^{4}$ que son fenómenos fuertemente asociados con la pobreza como también lo es el desempleo abierto.

El desempleo, subempleo, la distribución desigual del ingreso y de la riqueza se han convertido en verdaderos obstáculos para mejorar la situación de la pobreza en el Distrito Central, a la larga la problemática tiene un carácter estructural y por lo tanto no se puede resolver con simples medidas de tipo coyuntural o programas de gobierno que sólo lidian temporalmente con la situación puesto que cuando el programa llega a su término, la pobreza sigue latente.

\subsection{Violencia criminal en el Distrito Central}

La violencia criminal ha tomado un lugar importante en el análisis de la realidad nacional sobre todo porque sus manifestaciones tienden a ser cada día más crueles, los ataques no distinguen clase social, edad, sexo, condición socioeconómica, preferencia política o religiosa.

La violencia tiene su mayor manifestación en forma de delitos tipificados en el Código Penal y su mayor incidencia se presenta en los principales centros urbanos del país.

La pobreza es generadora de inseguridad e inestabilidad social, en una situación de pobreza, la presión para delinquir puede ser mayor, pero entre ser pobre y convertirse en delincuente, media una serie de situaciones que van desde el conformismo de pobre y marginado hasta los valores internalizados que pueden funcionar como una contra tendencia en una sociedad donde los medios de comunicación señalan a diario las carencias de la población e invitan a un mejor nivel de vida, a través de la obtención de bienes de consumo. Sin embargo, la oferta de bienes que hace el mercado no coincide con la oferta de empleo y salario que ofrece la estructura productiva y social (Castellanos, J 1997:7).

En un estudio realizado por el IUDPAS (2008) en relación a la percepción sobre la violencia e inseguridad en el Distrito Central evidenciada en forma de delitos refiere un aumento en este tipo de hechos, esta situación es crítica en el contexto de los delitos cometidos en la ciudad, de igual manera el escenario en los barrios y colonias no es nada halagador, puesto que el 54\% de la población consultada manifestó observar un incremento de los delitos en su barrio o colonia (Ver gráfico No. 3).

Entre las principales razones expuestas en relación al aumento o permanencia de los delitos están: el consumo de drogas $21.0 \%$, el desempleo/falta de trabajo con $14.3 \%$, el consumo de bebidas alcohólicas, $12.7 \%$ y la ineficiencia policial, 10.9\% (IUDPAS, 2008).

En relación a este último aspecto las personas que interponen sus denuncias ante la Policía Nacional Preventiva, La Dirección Nacional de Investigación y la Fiscalía muestran un alto grado de insatisfacción con el desempeño de estas instituciones, en general consideran que las autoridades no se interesan en el caso, o no hicieron lo suficiente por resolverlo, denotando incompetencia y falta de profesionalismo.

\subsection{Principales hechos que evidencian violencia cri- minal en el Distrito Central}

El incremento en los índices de violencia e inseguridad en la capital de la república son cada vez más evidentes, a diario los medios de comunicación concentran

Gráfico No. 3. Distrito Central: ¿Considera usted que en los últimos años el delito en la capital y donde usted vive ha aumentado, permanece igual o ha disminuido?

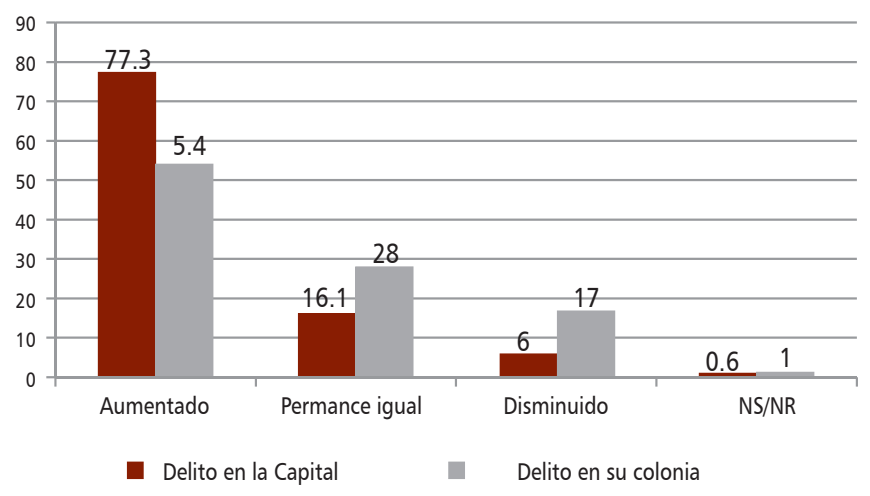

Fuente: IUDPAS, Percepción de inseguridad en el Distrito Central, 2008.

4. Subempleo visible Personas que trabajan menos de 36 horas semanales y que no trabajan más por no encontrar empleo. Subempleo invisible Personas que habiendo trabajado 36 horas semanales tuvieron ingresos inferiores al salario mínimo. 
gran parte de la información en dar a conocer la lista de delitos ocurridos a lo largo del día.

Los hechos delictivos que muestran violencia criminal en el Distrito Central son en su mayoría: robos, asaltos, homicidios, sicariato y los delitos conexos al narcotráfico y crimen organizado.

\section{- Robo y asaltos en el Distrito Central}

Los hechos delictivos como causa de violencia e inseguridad en el Distrito Central son los robos y asaltos ${ }^{5}$ que diariamente experimenta la población como víctima de la delincuencia común.

Durante el primer semestre del 2009 la Dirección General de Investigación Criminal de la Secretaría de Seguridad registró 3,878 denuncias por causa de robo y asalto en el municipio del Distrito Central, sin embargo, en gran parte de los casos, este tipo de hechos no es denunciado sobre todo porque la población considera que en pocas ocasiones se logra identificar a los responsables del delito. Un 40\% de los casos denunciados se hace porque el robo o asalto implica la pérdida de documentos personales y su reposición obliga a presentar la denuncia ante las autoridades competentes (DGIC, Planeamiento Estratégico, 2009).

La EPHPM aplicada en mayo del 2006, consideró un módulo de seguridad ciudadana. Los resultados permitieron captar estadísticamente la situación y percepciones de la población en relación al tema. En tal sentido, ante el delito de robo o asalto el 38\% de las personas encuestadas declaró haber sido víctima de este tipo de agresión. En otras palabras más de un tercio de la población capitalina sufrió de robo o asalto, en relación a la incidencia del delito atendiendo al sexo, fueron ligeramente más asaltadas o robadas las mujeres con 53\% (INE, EPHPM, 2006).

Las denuncias presentadas constataron que las zonas de la ciudad que ocupan el primer lugar de robos y asaltos son los barrios y colonias donde se ubican sectores de clase media, estos vecindarios se vuelven atractivos para los delincuentes. Si ingresan a las casas, las familias poseen bienes de valor que fácilmente pueden transportarse (joyas, laptop, cámaras, TV plasmas y dinero entre otros), y por otro lado, si el delito se comete en espacios públicos la probabilidad de que la población porte celulares caros, dinero en efectivo o tarjeta de crédito es mayor, de igual manera los robos que se dan en la calles y avenidas de estos vecindarios implican atraco a los vehículos estacionados; se roban baterías, llantas, vidrios, espejos, equipo de sonido, insignias, herramientas y en algunos casos se roba el vehículo completo.

El segundo lugar en denuncias por robo se presenta en los barrios del Centro Histórico de Tegucigalpa y Comayagüela. En el caso de los barrios de Tegucigalpa la incidencia predomina en Barrio el Centro y Barrio Abajo y en el caso de Comayagüela en la zona de los mercados. En relación a estos últimos las víctimas de robos y asaltos son los locatarios y los clientes que realizan sus compras.

Varias veces me han asaltado y he visto como al de enfrente también le han quitado el dinero que ha hecho durante el día, pero ¿qué vamos hacer con una pistola?, dígame, iquién se mueve?

Locatario del mercado Colón

Los clientes que realizan sus compras en los mercados de Comayagüela generalmente son los residentes de los barrios y colonias capitalinas pobres quienes aducen estar conscientes que arriesgan su seguridad al realizar sus compras en esa zona comercial de la capital, sin embargo, es allí donde encuentran los precios más cómodos y los productos más frescos.

Con lo poco que gano no puedo darme el lujo de acudir a los supermercados, aunque cada vez que voy al mercado tengo que arriesgar mi seguridad, lo que hago es encomendarme al Divino Creador para que me guarde y me proteja cada vez que realizo mis compras, pues no me queda de otra.

Doña Consuelo, usuaria de los mercados de Comayagüela

5. El robo es un delito contra el patrimonio, consistente en el apoderamiento de bienes ajenos, con intención de lucrarse, empleando para ello fuerza en las cosas o bien violencia o intimidación en la persona. El asalto se diferencia del robo porque involucra una confrontación personal entre individuos, la amenaza de uso de fuerza, o la propia utilización de la fuerza, junto con el propósito de coaccionar al otro y evitar cualquier resistencia. 
La Cuarta Estación Policial asigna a la zona de los mercados capitalinos diariamente 40 agentes policiales para que vigilen y custodien la vida de las personas que venden y compran sus productos en estos mercados, sin embargo, la delincuencia no parece mermar. Algunos consideran que existe complicidad entre los delincuentes y la policía por lo que la administración de algunos mercados ha decido contratar los servicios de vigilancia privada para resguardar las vidas y los bienes de los clientes y locatarios del lugar.

El tercer lugar en cuanto a denuncias por robo, corresponde a los barrios y colonias más pobres de la capital ubicados en su mayoría en la periferia de la ciudad en donde delincuentes comunes a diario agreden a la población mediante robo y asaltos; estos delitos se provocan especialmente en los espacios públicos de los vecindarios, sin embargo, no se descarta la invasión a la propiedad privada, se dan casos en los cuales hasta la ropa que esta secándose en los patios llega a ser hurtada.

Otro de los espacios de la capital que reporta el 15.6\% de las denuncias por robo son los espacios públicos del Distrito Central, especialmente calles, avenidas, parques y plazas, puntos de semáforos y paradas de transporte público. Frecuentemente las paradas en los semáforos son puntos estratégicos usados por los delincuentes comunes para robar las pertenencias sobre todo a las mujeres conductoras, a menudo suelen acercarse a la víctima especialmente si está lleva los vidrios abiertos, le amenazan con vocabulario grosero obligándole a entregar lo que se le solicita, incluso en el caso en que los vidrios van cerrados intentan abrir las puertas y en ocasiones llegan a amenazar con armas. Otro de los delitos comunes en los espacios públicos es el robo de teléfonos celulares; muchos capitalinos han perdido la vida en atracos de este tipo sobre todo porque se enfrentan a los delincuentes dejando saldos trágicos.

\section{- Homicidios en el Distrito Central}

Otro hecho delictivo que muestra violencia en el Distrito Central son los homicidios ${ }^{6}$
Para el 2008 la tasa de homicidio a nivel nacional fue de 58 por cada 100,000 habitantes. En el caso del municipio del Distrito Central se registró una tasa de 61 por cada 100,000, mayor a la presentada a nivel nacional. De los casos ocurridos en el departamento de Francisco Morazán el municipio del Distrito Central agrupa el 90.1\% de los homicidios por lo que este tipo de delito presenta una distribución netamente urbana (Observatorio de la Violencia, 2008). Esta es una de las situaciones que más preocupa en el municipio y ante la cual no se vislumbran soluciones efectivas.

El Observatorio de la Violencia del D.C. identifica los homicidios relacionados con riñas por causa de violencia doméstica e intrafamiliar, los relacionados con delincuencia común y delincuencia organizada, ajuste de cuentas vinculadas al narcotráfico y a la acción de las maras. Pero lo realmente preocupante es el hecho que la mayoría de los homicidios presenta una causa desconocida.

Los datos reflejan un incremento de homicidios por causa del crimen organizado, lamentablemente la mayoría de los crímenes queda en la impunidad debido a que las investigaciones no son efectivas.

La modalidad sicariato concentra el $36,2 \%$ esto incluye las muertes relacionadas con el crimen organizado, con la utilización de sicarios y en su totalidad cometidos con armas de fuego seguidas de muertes en robos que aportaron un 7.8\% y las riñas interpersonales con $5.5 \%$ de los casos, el resto de los móviles $4.6 \%$ está relacionado con delitos de violencia intrafamiliar, acción policial, maras, venganza, violación, extorsión y problemas de herencia, entre otros (IUDPAS, 2008).

Atendiendo a la distribución geográfica urbana de los homicidios, se identifica a los barrios de Comayagüela como los lugares de mayor peligro en relación a la ejecución de muertes por homicidio, en donde la zona de los mercados de Comayagüela, y la zona de El Carrizal reportan la mayor cantidad de muertes por homicidio. En

6. En el contexto de esta investigación está referida a las muertes violentas intencionales provocadas por un agresor, incluye también el homicidio culposo o negligente. 
el caso de los barrios de Tegucigalpa la colonia Villanueva ocupa el primer lugar (Ver gráfico No. 4).

Bajo el enfoque sociológico, la explicación de los homicidios según el área geográfica de ocurrencia estaría determinado por la pobreza y la falta de fuentes de empleo. Gran parte de los barrios que se muestran en el gráfico No. 4 representan las zonas más pobres de la capital en donde las desigualdades sociales y la falta de oportunidades son evidentes.

Esta situación hace reflexionar sobre las causas de la violencia e inseguridad en el Distrito Central las que se vinculan a factores estructurales de exclusión, desigualdad social, fragilidad institucional, acciones de narcotráfico y crimen organizado, pero también debe tenerse en cuenta los nuevos factores culturales que tienen que ver con las formas violentas de enfrentar los conflictos muchas de las cuales atentan contra el respecto a la integridad física de las personas y sus bienes.

- El narcotráfico de drogas y el crimen organizado en el Distrito Central.

El narcotráfico es una actividad que tomó notoriedad en Honduras a finales de la década de los setenta, sobre

\section{Gráfico No. 4. Distrito Central: muertes por homicidios, según barrios y colonias, período 2007- 2009}

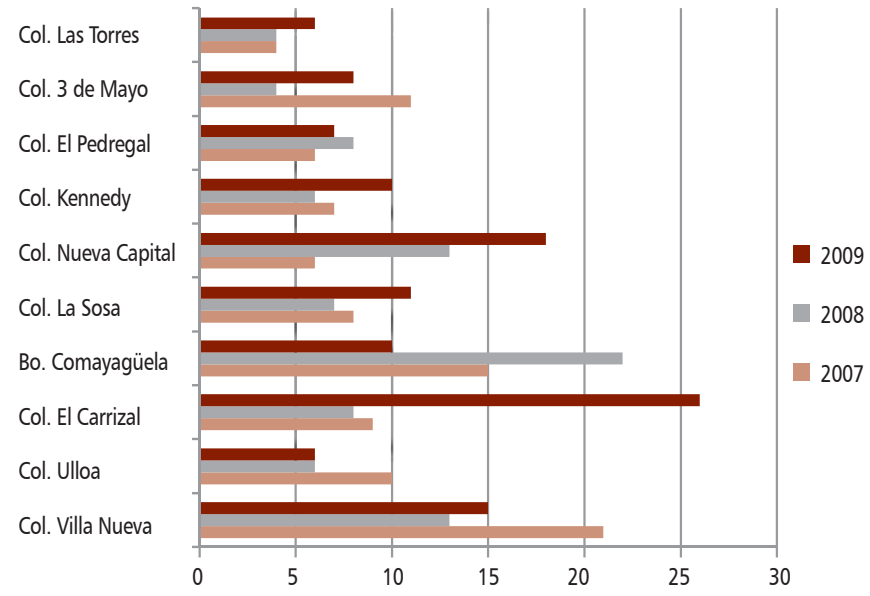

Fuente: Elaboración propia en base a los datos del Observatorio de la Violencia del Distrito Central, IUDPAS; 2007- 2009. todo a partir del asesinato de los esposos Ferrari, quienes de acuerdo a información policial eran traficantes de drogas además de tener vínculos con el tráfico de armas y de esmeraldas junto al hondureño Ramón Mata Ballesteros ${ }^{7}$, desde ese entonces se considera que el país era un punto de tránsito de la cocaína producida en América del Sur y cuyo destino principal era Estados Unidos de América.

Durante la primera década del siglo XXI y especialmente en los últimos 4 años (2007-2010) las actividades relacionadas con el narcotráfico se han incrementado en el país. Según especialistas, esto se debe a que Honduras además de ser un país de paso para la droga también se convirtió en un país de consumo.

En el caso particular de la capital de la república al concentrar un porcentaje significativo de la población se vuelve un importante mercado para la venta y consumo de drogas como la marihuana, la cocaína y el crack entre las de mayor circulación. Muchos de los barrios y colonias capitalinas son mercados del narcotráfico en pequeña y mediana escala. El consumo de drogas ilegales en la capital ha tenido un notable crecimiento entre la población, es conocido que éstas se venden en sitios de recreación como discotecas, bares, parques y plazas de la ciudad, así como en las calles de barrios y colonias capitalinas. Los consumidores del producto no distinguen estrato social, igual consumen los estratos altos, medios y bajos por razones diferentes.

Muchos vecindarios se han vuelto mercados del narcotráfico en pequeña escala; para el caso es común encontrar quien venda un carruco de marihuana por la cantidad de 10.00 Lempiras (\$0.50), como es común que gran parte de población que lo consume sean jóvenes, sin embargo, personas de todas las edades se encuentran atrapadas en este tipo de vicio.

En relación a la venta de drogas ilícitas son muchas las personas involucradas, en algunos casos quizá la pobreza, la falta de empleo, la falta de seguridad social conduce a realizar este tipo de actividades ilícitas. En la

7. Hondureño, reclamado por los delitos de narcotráfico y homicidio en México, Estados Unidos, Colombia y Honduras. Se fugó de varias cárceles de alta seguridad. Entre ellas EGLIN (EE.UU.), LA PICOTA y MODELO (Colombia). El convicto se radico en Honduras, después de haberse fugado de una cárcel de máxima seguridad de Colombia, estando en nuestro país, se mencionó que le ofreció al Presidente José Azcona Hoyo pagar la deuda externa de Honduras. www.angelfire.com 
capital de la república se dan varios casos en los cuales hombres, mujeres y hasta personas de la tercera edad han sido capturados de manera infraganti vendiendo carrucos de marihuana y piedras de crack a quien se lo solicite.

Una de las razones que explica el incremento de las actividades del narcotráfico en el país es la guerra que el gobierno mexicano le ha declarado a los carteles de la droga provocando que estas estructuras trasladen sus centros de operaciones a Centroamérica. En el caso de Honduras, este tipo de actividad ha incrementado los niveles de violencia.

El aumento de consumo de drogas es preocupante, también alarma todos los delitos conexos al narcotráfico que incluye el ajuste de cuentas, el sicariato y el lavado de dinero, actividad que encubre las actividades del narcotráfico mediante el surgimiento de negocios de aparente legalidad y aunque en Honduras existe una Ley contra el Delito de Lavado de Activos, aprobada mediante Decreto No.45-2002 muy pocas veces se aplica.

El lavado de activos es el brazo más fuerte de los cárteles de la droga, pero en Honduras es un delito que casi no es perseguido por lo que el país es considerado prácticamente un paraíso para los que se dedican a esa ilícita actividad.

www.laprensa.hn/Series/content/view/section/14186

En relación al ajuste de cuentas y sicariato estos delitos han tomando fuerza en los últimos años dejando como resultado la muerte de gran número de oficiales de policía, abogados y periodistas; de igual manera se han asesinado muchas personas de diferentes actividades profesionales o laborales.

Gran parte del sicariato y ajuste de cuentas se liga a la lucha entre carteles de la droga por el dominio de territorios o mercados y a conflictos en la rendición de cuentas, para el caso hay personas que adquieren deudas o no las rinden cabalmente y como los negocios relacionados con el mundo de la droga son hechos ilícitos los conflictos generados al interior no se pueden llevar a los tribunales, éstas se cobran matando a la persona que es deudor o que ha fallado en el negocio.

Respecto a los ejecutores de estos delitos se considera que los sicarios son personas entrenadas que utilizan generalmente como medio de transporte las motos con las que se desplazan rápidamente por las calles huyendo fácilmente de la escena del crimen. El ingeniero Armando Calidonio Viceministro de Seguridad en la primera parte del mandato de Porfirio Lobo Sosa, considera que el sicariato surge ante la necesidad del narcotráfico de tener un brazo armado, en ese sentido, se reclutan personas que son entrenadas para matar. El costo de estos asesinatos varía y dependen del riesgo y la capacidad económica del contratante. Según informes policiales, en la capital hay matones que cumplen misiones hasta por 500 ó 1,000 Lempiras (\$26.00-52.00). Se conoce que algunos tasan el precio de acuerdo con la persona a la que hay que aniquilar.

Generalmente los sicarios usan moto y trabajan en pareja uno conduce y otro dispara a la víctima, en algunos casos se usan vehículos, generalmente utilizan pistolas 9 milímetros pero hay ocasiones en las que se utilizan armas de grueso calibre.

Este año, la industria del sicariato ha dado un giro inesperado. Mientras en las administraciones pasadas las víctimas eran personas ligadas al crimen organizado, robo de vehículos o narcotráfico, las últimas víctimas han sido operadores de justicia, abogados o comunicadores.

El Heraldo, 07-03-10

A raíz del incremento de crímenes a manos de sicarios y ante la coyuntura que éstos se han consumado en su mayoría desde motocicletas en donde el autor material ha sido el copiloto se pretendió implementar medidas restrictivas que contemplaran la prohibición de transportar dos hombres a la vez en una motocicleta, en tanto se implementaba el uso de chaleco y un casco numerado, tratando de reducir el accionar de los delincuentes en motocicletas, sin embargo, a la fecha no se han implementado éstas medidas.

"Lo que estamos buscando es cerrar espacios a los delincuentes, que la motocicleta no sea una herramienta de utilización para los sicarios o, por lo menos, que lo piensen dos veces".

Oscar Álvarez, Secretario de Seguridad, El Heraldo 9/04/2010 
Aunque los últimos gobiernos han intentado controlar las actividades del narcotráfico y los delitos conexos sus esfuerzos no han sido exitosos y la lucha contra el narcotráfico se está perdiendo, prueba de ellos es el asesinato que sufrió por parte de sicarios el titular de la Dirección de Lucha contra el Narcotráfico de Honduras General Julián Arístides González, ocurrido en el Barrio Guanacaste, frente a una escuela de educación primaria a las 6.50 de la mañana. A juzgar por los hechos la policía está perdiendo la guerra frente a la actividad delincuencial.

\section{Conclusiones}

- En el Distrito Central la violencia estructural se refleja en el acceso diferenciado de amplios sectores de la población capitalina a servicios básicos como agua potable, alcantarillado sanitario, educación, y una vivienda construida con materiales de calidad.

- El MNBI refleja que más de la tercera parte de la población ha sufrido violencia estructural hecho evidente en el deterioro de las condiciones de vida producto en alguna medida del impacto del modelo económico neoliberal que conlleva efectos negativos sobre la inversión del Estado en gastos sociales y sobre el mercado de empleo urbano en particular para los trabajadores de menor calificación que deben aceptar ocupaciones precarias, informales con baja remuneración. Esta situación genera limitadas opciones en mejoramiento de su vivienda y en general de su nivel de bienestar. La situación de violencia estructural en el Distrito Central en el contexto de la LP, muestra que más de la mitad de la población capitalina enfrenta condiciones de pobreza esto limita significativamente sus posibilidades de desarrollo.

- En el plano de la violencia criminal son cada vez más frecuentes los delitos registrados por las autoridades competentes entre los cuales prevalecen aquellos ligados a robos y asaltos, homicidios, las actividades del narcotráfico y crimen organizado que opera en todas las esferas de la sociedad y cuyas redes se han extendido considerablemente, a tal grado que la venta y comercialización de drogas es una actividad cotidiana que se registra día a día en gran parte de los barrios y colonias capitalinas en detrimento de la salud física y mental de la población.

- Actualmente muchas de las políticas de Estado están encaminadas a frenar la violencia criminal, se trata de abordar el tema de seguridad bajo un concepto integral que tenga en cuenta las diversas esferas de la vida humana y que genere oportunidades entre los más desposeídos de la sociedad.

\section{Bibliografía}

- Castellanos, J (1997). La Inseguridad Ciudadana, $\mathrm{CEDOH}$, Documento de Análisis Boletín Especial No. 79, Honduras.

- DGIC (2009) Oficina de Planeamiento Estratégico, Tegucigalpa, Honduras.

- Diario El Heraldo (2010), Tegucigalpa 7 de Marzo.

- Diario El Heraldo (2010), Tegucigalpa 9 de Abril.

- Guerrero V, Rosa María (2006) Nosotros y los Otros: Segregación Urbana y Significados de la Inseguridad en Santiago de Chile, en Lugares e Imaginarios en la Metrópolis, Coordinación Daniel Hiernaux, Editorial Anthopos, UAM, México.

- INE (2002-2009) Encuestas Permanentes de Hogares de Propósitos Múltiples, mayo, Honduras, C.A.

- Jiménez Bautista, F, Muñoz, Adolfo (2004) Violencia Estructural en Mario López Martínez (dir.), et al. Enciclopedia de Paz y Conflictos: L-Z. Edición especial.

- Luna, Víctor Manuel (2005) Criticas al Interés del Banco Mundial por la Pobreza. Ponencia Primer Encuentro Internacional sobre Pobreza, Desigualdad y Convergencia del 3 al 30 de marzo.

- IUDPAS-UNAH (2009) Diagnóstico sobre Inseguridad Ciudadana en el Distrito Central, Tegucigalpa.

- IUPDPAS-UNAH (2007-2009) Boletín Observatorio de la Violencia del Distrito Central. Mortalidad, Tegucigalpa, Honduras.

- www.angelfire.com

- www.laprensa.hn/Series/content/view/section/14186. 